\title{
Improved Canny Edges Using Cellular Based Particle Swarm Optimization Technique for Tamil Sign Digital Images
}

\author{
M. Krishnaveni, P. Subashini, T.T. Dhivyaprabha \\ Department of Computer Science, Avinashilingam Institute for Home Science and Higher Education for Women, India
}

\begin{tabular}{l}
\hline \hline Article Info \\
\hline Article history: \\
Received May 9, 2016 \\
Revised Jul 7, 2016 \\
Accepted Jul 17, 2016 \\
\hline
\end{tabular}

\section{Keyword:}

Canny edges

Cellular organism

Particle swarm optimization

Tamil sign language

Thresholding

\begin{abstract}
The development of computer based sign language recognition system, for enabling communication with hearing impaired people, is an important research area that faces different challenges in the pre-processing stage of image processing, particularly in boundary detection stage. In edge detection, the possibility of achieving high quality images significantly depends on the fitting threshold values, which are generally selected using canny method, and these threshold values may vary, based on the type of images and the applications chosen. This research work presents a novel idea of establishing a hybrid particle swarm optimization algorithm, which is a combination of PSO with the behavioural pattern of cellular organism in canny method that defines an objective to find optimal threshold values for the implementation of double thresholding hysteresis method, which is viewed as a non-linear complex problem. The attempt to incorporate the model has minimized the problem of quick convergence of PSO algorithm which has improved the detection of broken edges. The efficiency of the proposed algorithm is proved through the experimental observation, done in Tamil sign images to indicate the better performance of canny operator by introducing new variant based PSO.
\end{abstract}

Copyright (C) 2016 Institute of Advanced Engineering and Science. All rights reserved.

\section{Corresponding Author:}

M. Krishnaveni, Department of Computer Science, Avinashilingam Institute for Home Science and Higher Education for Women, Coimbatore-641043, Tamil Nadu, India.

Email: krishnaveni.rd@gmail.com

\section{INTRODUCTION}

Sign language is the only reliable tool for understanding skills among the deaf people. Also, the proficient activities of hearing impaired people can be improved only by using consistent SLR system. But there is no single standard form of sign language, and it varies from region to region. Tamil Sign Language (TSL) is a region based sign language, considered to be a more useful means to have confined improvement in their own boundary [1]. This language for deaf people is gaining factual importance in the regional communication. The main objective of this paper is to propose a robust edge detection method, using hybrid optimization technique, which could be a fine contribution for automated system that identifies and recognizes the TSL signs. As the first step, the image acquired, using digital images, are resized into 256x256 and converted into grayscale image and that image is preprocessed by optimized weighted median noise filtering technique [2]. As a primary analysis of segmentation phase, common edge recognition algorithms such as Sobel, Robert, Canny and Prewitt are experimented, for which the scope of canny is extended, by introducing PSO, and that PSO is improvised by incorporating the motility behaviour of cellular organism called a new variant Cellular Particle Swarm Optimization (CPSO) algorithm. The experiments are carried out with 18 Tamil consonants and each represents the Static images of the palm side of right hand. Figure 1 depicts the generated consonants of Tamil signs that belong to TSL dataset. The organization of the paper is as follows: Section 2 introduces over edge preserving details through thresholding concepts, using canny 
operator. Section 3 explains the proposed hybrid canny edge detection method using PSO, by introducing a new variant. Section 4 illustrates the results and analysis, based on evaluation assessments of the experimental results. Section 5 concludes the findings and the scope of the research work in TSLR.

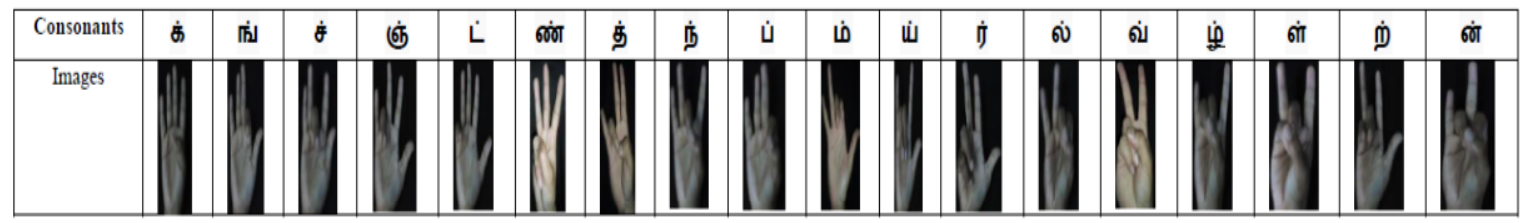

Figure 1. Manually Generated Tamil Sign Language Dataset (consonants)

\section{BOUNDARY DETECTION USING THRESHOLDING}

An edge is defined based on the swift change of intensity of an image. Edge detection is a process of finding the sharp contrast based on the intensities of an image, by reducing the amount of data in an image, while preserving important structural features of that image [3]. The methods for detecting the edges depend on the computation of image gradients and the type of filter used to calculate gradient estimates in the horizontal and vertical directions. Therefore, the threshold value is the one which decides whether edges are present or not at an image point [4]. The crucial problem is the issue of choosing the thresholds. A commonly used method for finding the appropriate threshold is canny method, in which the resultant output contains thin edges, and the edge pixels are linked using edge tracking procedure with hysteresis thresholding method [5]. It varies the threshold by tracking the edge once and finding the calculated threshold as the second resultant argument. This method is therefore better at suppressing noise, and more likely to detect true weak edges [6]. Therefore, the operator finds its design to be best edge detector. For implementation, the discrete approximation to Gaussian function is done with $\sigma=1.4$, and the Sobel operator is performed to find the approximate absolute gradient magnitude at each point with the convolution of mask $\mathrm{G}_{\mathrm{x}}$ and $\mathrm{G}_{\mathrm{y}}$.

\begin{tabular}{|c|c|c|c|c|c|c|c|c|c|c|c|c|c|}
\hline \multirow{5}{*}{$\begin{array}{c}\text { Discrete } \\
\text { approximation } \\
\sigma=1.4 .\end{array}$} & 2 & 4 & 5 & 4 & 2 & \multirow{4}{*}{$\mathbf{G}_{\mathbf{x}}=$} & \multirow{4}{*}{\begin{tabular}{|l|}
-1 \\
-2 \\
-1 \\
\end{tabular}} & \multirow{4}{*}{$\begin{array}{l}\mathbf{0} \\
\mathbf{0} \\
\mathbf{0}\end{array}$} & \multirow{3}{*}{$\frac{1}{2}$} & \multirow{4}{*}{$\mathbf{G}_{\mathbf{y}}=$} & \multirow{3}{*}{$\frac{1}{0}$} & \multirow[b]{2}{*}{2} & \multirow[b]{2}{*}{1} \\
\hline & 4 & 9 & 12 & 9 & 4 & & & & & & & & \\
\hline & 5 & 12 & 15 & 12 & 5 & & & & & & & 0 & 0 \\
\hline & 4 & 9 & 12 & 9 & 4 & & & & 1 & & -1 & -2 & -1 \\
\hline & 2 & 4 & 5 & 4 & 2 & & & & & & & & \\
\hline
\end{tabular}

However, this problem of choosing appropriate threshold values may vary over the image [6]. Therefore, to improve the canny edges, this paper proposes to find the optimal threshold values, by implementing nature-inspired computing technique, in combination with behavioural pattern of cellular organism.

\section{IMPROVED CANNY EDGES USING PROPOSED CELLULAR PSO}

Though the appropriate threshold values is chosen in canny method, the edge details that have to be preserved are more in sign images, and they vary according to different images. The proposed method defines an objective to choose the optimal threshold values, using CPSO, which is also compared with classical PSO-based canny edge detection [7]. In this research work, the proposed CPSO algorithm is applied in the canny method, that defines an objective, to find optimal threshold values for the implementation of double thresholding hysteresis method, which is viewed as a non-linear complex problem [8].

\subsection{Particle swarm optimization with fibroblast behaviour}

Particle Swarm Optimization (PSO) is a nature inspired algorithm proposed by Kennedy and Eberhart (1995). This algorithm is developed by the characteristics observed from birds flocking and fish schooling. Each particle $\left(\mathrm{p}_{\mathrm{i}}\right)$ has its own position $\left(\mathrm{x}_{\mathrm{i}}\right)$ and velocity $\left(\mathrm{v}_{\mathrm{i}}\right)$ in the evolutionary region. A set of parameters such as inertia weight $(\omega)$, constriction coefficients $(K)$, acceleration factors $\left(\varphi_{1}, \varphi_{2}\right)$, velocity range [-vmax,+vmax] and random numbers $\left(\mathrm{r}_{1}, \mathrm{r}_{2}\right)$ enforce the swarm to migrate in the $\mathrm{n}$ - dimensional problem space, and evaluated using the fitness function until the predetermined criteria (s) or the maximum

Improved Canny Edges Using Cellular Based Particle Swarm Optimization Technique .... (M. Krishnaveni) 
number of iterations $(\mathrm{t})$ is met. The objective is to find optimal solution (individual best $\left(\mathrm{P}_{\text {best }}\right)$ and social best $\left.\left(\mathrm{G}_{\text {best }}\right)\right)$ by updating the velocity $\left(\mathrm{v}_{\mathrm{i}}\right)$ coordinates to position $\left(\mathrm{x}_{\mathrm{i}}\right)$ equations over time which are represented in eq. 1 and eq. 2 [9].

$$
V_{i j}^{(t+1)}=\omega * K * V_{i j}^{(t)}+\left(\varphi_{1} * r_{1} *\left(P_{b e s t}-x_{p}^{(t)}\right)\right)+\left(\varphi_{2} * r_{2}\left(P_{g}-x_{p}^{(t)}\right)\right)
$$

where

$$
\begin{aligned}
& K=\frac{2}{\mid 2-\varphi+\varphi \sqrt{\varphi^{2}-4 \varphi}}, \varphi=\varphi_{1}+\varphi_{2} \\
& \omega=0.5-\frac{\operatorname{rand}()}{2}
\end{aligned}
$$

rand () is a random function that generates a distributed random number which lies within a range of 0 and 1.

$$
X_{i j}^{(t+1)}=X_{i j}^{(t)}+V_{i j}^{(t+1)}
$$

In a few attempts, it is observed that the quick convergence of PSO algorithm cannot find multiple optimal solutions in a single search space problem. This intricacy is controlled by incorporating the motility behavior of fibroblast organism [10]. The motility factor $(\sigma)$ acts as an energetic force that enables the swarm to proliferate around the entire problem space and the particles are accelerated towards the best candidate solutions which have better fitness values. The functionality of swarm has been progressed with migration factor $(\sigma)$ which is applied in neighborhood particles that increases the degree of interaction among the population [11]. Henceforth, the randomly generated collagen enhanced the moving speed of particles, and the efficiency of CPSO algorithm to obtain optimal solution has been improved. The algorithm 1 gives the steps for implementation of CPSO technique.

\section{Algorithm 1. Cellular Particle Swarm Optimization (CPSO)}

Step 1: Initialize the particles of population size $M_{i}(i=1,2, \ldots, n)$ with randomly generated position $x_{i}$ and velocity $v_{i}$ in the $n$-dimensional search space.

\section{Begin}

\section{Step 2: Repeat}

Evaluate the objective function of every particle using standard benchmark function.

Step 3: Compare the fitness value of each particle $F\left(\mathrm{M}_{\mathrm{i}}\right)$ with the value of individual best $\mathrm{P}_{\text {best }}$. If the current value of a particle $F\left(M_{i}\right)$ is better than $P_{\text {best }}$, then the value of $M_{i}$ is set to $P_{\text {best }}$ and then the position of a current particle $\mathrm{x}_{\mathrm{i}}$ is assigned to $\mathrm{P}_{\mathrm{i}}$ in the problem space.

Step 4: Identify the neighborhood best $\left(\mathrm{G}_{\text {best }}\right)$ particle in the population. If the current value of a particle $F$ $\left(\mathrm{M}_{\mathrm{i}}\right)$ is better than $F\left(\mathrm{G}_{\text {best }}\right)$, then the value of $\mathrm{M}_{\mathrm{i}}$ is set to $\mathrm{G}_{\text {best }}$. The index value of a current particle $\mathrm{x}_{\mathrm{i}}$ is assigned to $\mathrm{P}_{\mathrm{g}}$.

Step 5: Update the velocity and position of particle according to the following equation:

$$
\begin{aligned}
& { }_{i j}^{(t+1)}=\omega * K * V_{i j}^{(t)}+\left(\varphi_{1} * R_{1} *\left(P_{b e s t}-x_{p}{ }^{(t)}\right)+\sigma *\left(\varphi_{2} * R_{2} *\left(G_{b e s t}-p_{g}{ }^{(t)}\right)\right)\right. \\
& X_{i j}^{(t+1)}=X_{i j}{ }^{(t)}+V_{i j}^{\left({ }^{(t+1)}\right.}
\end{aligned}
$$

where

$$
\mathrm{V}_{\mathrm{ij}}^{(\mathrm{t})}=\text { velocity of } \mathrm{j}^{\text {th }} \text { particle in } \mathrm{i}^{\text {th }} \text { iteration at time } \mathrm{t}
$$

$\mathrm{X}_{\mathrm{ij}}{ }^{(\mathrm{t})}=$ position of $\mathrm{j}^{\mathrm{th}}$ particle in $\mathrm{i}^{\text {th }}$ iteration at time $\mathrm{t}$

$\mathrm{R}_{1}, \mathrm{R}_{2}=$ random numbers lies between 0 and 1

$\mathrm{G}_{\text {best }}=$ Neighborhood (social) particle best $\mathrm{g}$

$\mathrm{x}_{\mathrm{P}, \mathrm{g}_{\mathrm{g}}}=$ Index value of current and neighborhood particle

$$
\begin{aligned}
& K=\frac{2}{\mid 2-\varphi+\varphi \sqrt{\varphi^{2}-4 \varphi}}, \varphi=\varphi_{1}+\varphi_{2} \\
& \sigma=\text { motility factor } \\
& \sigma=\psi a^{2} e^{-a / \text { asat }}
\end{aligned}
$$


where

$$
\begin{aligned}
& \psi=10^{4}-1 \\
& \mathrm{a}_{\text {sat }}=1.1 \\
& a=\left[\frac{d\|c\|}{d t}\right]=\left(p_{c-d_{c}}|| c||\right) \sum_{i=1}^{M} \omega\left(f^{i}-x\right)
\end{aligned}
$$

where

$$
\begin{aligned}
& p_{c} \text { and } d_{c} \text { are positive constants } \\
& \|c\|=\text { from } 0 \text { to } 0.208 \\
& \|\omega\|=\text { from } 0.33 \text { to } 1 \\
& \mathrm{f}^{i}=\mathrm{i}^{\text {th }} \text { direction of a vector } \mathrm{f} \\
& \mathrm{x}=\text { position of a particle }
\end{aligned}
$$

Until termination condition(s) / maximum iteration (s) is met.

\section{End}

\subsection{Cellular based PSO for improved canny edges}

Cellular particle swarm optimization algorithm is implemented to find the optimal solutions (low L and High $\mathrm{H}$ ) that can be applied for segmentation of the regional sign language images. The ranges of thresholds are estimated through histogram analysis of the sign language dataset. The procedure of CPSO optimized canny method to perform edge detection is described below in algorithm 2 .

\section{Algorithm 2:}

Step 1: Input a regional sign language images I $(\mathrm{u}, \mathrm{v})$.

Step 2: Smoothing - Blurring of the image to remove noise by applying a Gaussian filter. The convolution of an image with a core of Gaussian filter using standard deviation of $\sigma=1.4$ is shown in equation (5) given below:

$$
B=\frac{1}{159}[24542 ; 491294 ; 51215125 ; 491294 ; 24542 ;]
$$

Step 3: Determination of gradients - Find edges by determining gradients of the input images to identify the varied intensity of the image. Gradients at each pixel have found using Sobel operator. It can be implemented to approximate the gradient in the $\mathrm{x}$ - and $\mathrm{y}$ - directions respectively in the smoothed image, by applying the kernels given in equation (6) and (7)

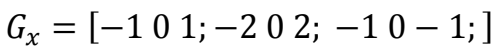

$$
\begin{aligned}
& G_{y}=\left[\begin{array}{llllll}
1 & 2 & 1 ; & 0 & 0 & 0 ;-1-2-1 ;
\end{array}\right]
\end{aligned}
$$

where, $\mathrm{G}_{\mathrm{x}}$ and $\mathrm{G}_{\mathrm{y}}$ are gradients in the $\mathrm{x}$ and $\mathrm{y}$ directions respectively;

The gradient magnitudes can be determined by applying an Euclidean distance measure, using the Pythagoras law as shown in equation (8).

$$
|G|=\sqrt{G_{x}^{2}+G_{y}^{2}}
$$

Step 4: Non-maxima suppression -A gradient image has blurred edges, which is transformed into sharp edges, by preserving all local maxima and eliminating other pixels in the image. This process consists of the following three steps:

- The gradient direction $\theta$ is rounded off to the nearest $45^{\circ}$, it corresponds to the 8 connected neighbourhood pixel values of the image,

- Compare the gradient magnitude of the current pixel value with the gradient magnitude of the pixels found in both positive and the negative direction, and

- If the gradient magnitude of the current pixel is largest, then preserve the value of the current pixel to improve the edge strength of the image. Otherwise, the pixel values are suppressed in the given gradient images.

Step 5: Double thresholding - The resultant edge pixels may contain noise or irrelevant values. The canny method uses double thresholding (high T1 and low T2) values to further suppress the noise content as well as preserve the true image. Edge pixels (T) larger than high threshold (T1) are marked as strong. Edge pixels (T) smaller than low threshold (T2) are removed. Edge pixels (T) that fall between T1 and T2 are considered as weak. Selection of threshold values (T1 and T2) can be considered as a nonlinear complex problem. Here, 
Cellular Particle Swarm Optimization (CPSO) algorithm is implemented to choose thresholds for a given regional sign language images. Procedure 1 and 2 are done to achieve step 5.

Step 6: Tracking of edges in the chosen images can be performed by using hysteresis thresholding technique - The segmented image is obtained as final output image and it is used for further analysis.

\section{Procedure 1: To find the range of threshold values using histogram method:}

Step 1:A regional sign language image dataset is given as input and the edges of sign language images are predetermined as Region of Interest (ROI) in the dataset.

Step 3: Input image is converted into gray scale image to reduce the size of the image.

Step 4: Repeat : Identify the histogram h (z) of an image to be segmented.

Step 5: The probability of a pixel value is represented in the equation (9):

$$
\begin{aligned}
& P(z)=p(z / \text { background pixel) } P(\text { background pixel })+p(\text { z/object pixel) } P \text { (object pixel) or } \\
& P(z)=P_{b} \frac{1}{\sqrt{2 \pi \sigma_{b}}} e-\frac{\left(z-\mu_{b}\right)^{2}}{2 \sigma_{b}^{2}}+P_{o} \frac{1}{\sqrt{2 \pi \sigma_{o}}} e^{-\frac{\left(z-\mu_{o}\right)^{2}}{2 \sigma_{o}^{2}}} \quad \text { or } \\
& P(z)=P_{b} p_{b}(z)+P_{o} p_{o}(z)
\end{aligned}
$$

where

$p_{b}(z), p_{o}(z)$ - probability distributions of background and object pixels

$\mu_{b}, \mu_{o}$ - means of the distributions

$\sigma_{b}, \sigma_{o}$ - standard deviations of the distributions

$P_{b}, P_{o}$ - a-priori probabilities of background and object pixels.

Step 6: The probability of misclassification of an object pixel as background is expressed in eqn (10):

$$
E_{o}(T)=\int_{-\infty}^{T} p_{o}(z) d z
$$

Step 7: The mathematical equation for the probability of background pixels, incorrectly classified as object pixels, is represented in eqn. 11 :

$$
E_{b}(T)=\int_{T}^{\infty} p_{b}(z) d z
$$

Step 8: The mathematical formula for threshold selection is obtained by minimizing the above expression as denoted in eqn. 12 :

$$
T=\frac{\mu_{b}+\mu_{o}}{2}
$$

Step 9: Until the threshold values is obtained for the images found in regional sign language dataset.

\section{Procedure2 : To find optimal threshold values (Low $L$ and High $H$ )}

Step 1: Initialization of swarm and parameters - A population of threshold particles says $\mathrm{p}$, of size $\mathrm{m}$ $(0.0<=\mathrm{n}<=1.0)$ are generated with random position $\mathrm{x}_{\mathrm{p}}$ and random velocity $\mathrm{v}_{\mathrm{p}}$.

Step 2: Fitness evaluation - Each particle is evaluated with standard benchmark function (griewank) which has continuous, differentiable, non-separable, scalable and multimodal properties for choosing the optimal threshold values. The mathematical equation of griewank function is represented in eqn.13.

$$
f(x)=\sum_{i=1}^{n} \frac{x_{i}^{2}}{4000}-\Pi \cos \left(\frac{x_{i}}{\sqrt{i}}\right)+1
$$

Step 3: Find the social best particle - Based on the evaluation of fitness function (minima), a neighborhood best (Social) $g_{b e s t}$ is chosen for each iteration.

Step 4: Compare the previous value $\mathrm{g}_{\text {besti-1 }}$ of the particle with the current particle $\left(\mathrm{g}_{\text {best }}\right)$ value.

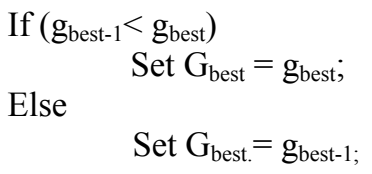


Step 5: Find the social best particle - Identify the neighbourhood (social) particle in the population and assign to index variable $\mathrm{g}$.

Step 6: Velocity and position updating equation - The velocity and position of a particle are updated using the following mathematical given in eqn. 14 and 15 :

$$
\begin{aligned}
& V_{i j}^{(t+1)}=\omega * K * V_{i j}^{(t)}+\left(\varphi_{1} * R_{1} *\left(P_{b e s t}-x_{p}{ }^{(t)}\right)+\sigma *\left(\varphi_{2} * R_{2} *\left(G_{b e s t}-p_{g}{ }^{(t)}\right)\right)\right. \\
& X_{i j}^{\left({ }^{(t+1)}\right.}=X_{i j}^{(t)}+V_{i j}^{(t+1)}
\end{aligned}
$$

where

$\mathrm{V}_{\mathrm{ij}}=$ velocity of $\mathrm{j}^{\text {th }}$ particle at $\mathrm{i}^{\text {th }}$ iteration

$\mathrm{X}_{\mathrm{ij}}=$ Position of $\mathrm{j}^{\text {th }}$ particle at $\mathrm{i}^{\text {th }}$ iteration

$\omega=0.729$

(i.e) random ( ) function generate a distributed random number which lies from 0 to 1

$\phi_{1}=\phi_{2}=2.0$

$\mathrm{R}_{1}, \mathrm{R}_{2}=$ random number lies between 0 and 1

$\mathrm{P}_{\text {best }}=$ Personal (individual) best

$\mathrm{G}_{\text {best }}=$ Neighbourhood (social) particle best,$\sigma=$ motility factor

Step 7: Repeat the steps from 3 to 7 either the maximum number of iterations or predetermined conditions is to be met.

Step 8: Continuous evolution of swarm in problem space - Cellular Particle Swarm Optimization (CPSO) algorithm offer $\mathrm{G}_{\text {best }}$ candidate solution (low and high threshold values) after the fitness execution of 10000 iterations.

Step 9: The resultant thresholds (high $\left[\mathrm{T}_{1}\right]$ and low $\left[\mathrm{T}_{2}\right]$ ) are applied for tracking of edges in regional sign language image dataset by using hysteresis thresholding.

This effort has ultimately improved the temperament of PSO algorithm to yield high quality results in sign images. The subjective evaluation result of canny method,PSO based canny and CPSO based canny are given in Figure 2.

\section{RESULTS AND ANALYSIS}

The experimental observation is demonstrated to indicate how the new variant algorithm outperforms the Classical PSO on all evaluated metrics. The experimental parameters set were the same for both PSO and CPSO. The particle size was set to 101 and the inertia weight was set to 0.729 . The execution of both PSO and CPSO were terminated with 10000 fitness evaluation iterations.

This effort has ultimately improved the temperament of PSO algorithm to yield high quality results in sign images. The subjective evaluation result of canny method, PSO based canny and CPSO based canny are given in Figure 2.

The proposed work is implemented in MATLAB and Java and it is tested in manually generated Tamil Sign Language (TSL) datasets with limitation of single handed signs and black background images. Each sign is captured with ten different signers. It is seen that cellular PSO has found better average solutions than traditional PSO based on similarity index and pearson correlation coefficient metrics for which the eqn (16) and (17) which are given below and the comparison of the results is shown in Table 1.

$$
\text { Similarity Index SSIM }=\frac{\left(2 \mu_{x} \mu_{y}+C_{1}\right)\left(2 \sigma_{x y}+C_{2}\right)}{\left(\mu_{x}^{2}+\mu_{y}^{2}+C_{1}\right)\left(\sigma_{x}^{2}+\sigma_{y}^{2}+C_{2}\right)}
$$

where, $\mu_{\mathrm{x}}=$ mean of $\mathrm{x}, \mu_{\mathrm{y}}=$ mean of $\mathrm{y}$ and $\mathrm{c}_{1}=\left(\mathrm{k}_{1} * \mathrm{~L}\right)^{2}{ }^{\prime} \mathrm{c}_{2}=\left(\mathrm{k}_{2} * \mathrm{~L}\right)^{2}$

$\mathrm{K}_{1}=0.01 ; \mathrm{k}_{2}=0.03$ by default, $\mathrm{L}=$ the dynamic range of the pixel values; By default $\mathrm{L}=255$

$\sigma_{x}^{2}=$ variance of $\mathrm{x}, \sigma_{y}^{2}=$ variance of $\mathrm{y}, \sigma_{\mathrm{xy}}=$ covariance of $\mathrm{x}$ and $\mathrm{y}$

$$
\text { Pearson Coefficent } r=\frac{\Sigma_{i}\left(x_{i}-x_{m}\right)\left(y_{i}-y_{m}\right)}{{\sqrt{\Sigma_{i}\left(x_{i}-x_{m}\right)}}^{2}{\sqrt{\Sigma_{i}\left(y_{i}-y_{m}\right)}}^{2}}
$$

where, $x_{i}=$ intensity values of $i^{\text {th }}$ pixel in image $x, y_{i}=$ intensity values of $i^{\text {th }}$ pixel in image $y, x_{m}=$ mean intensity values of image $\mathrm{x}, \mathrm{y}_{\mathrm{m}}=$ mean intensity values of image $\mathrm{y}$. When there is a value of $r=1$, if the two images are absolutely identical, $r=0$ if they are completely uncorrelated and $r=-1$ if they are completely anticorrelated. 


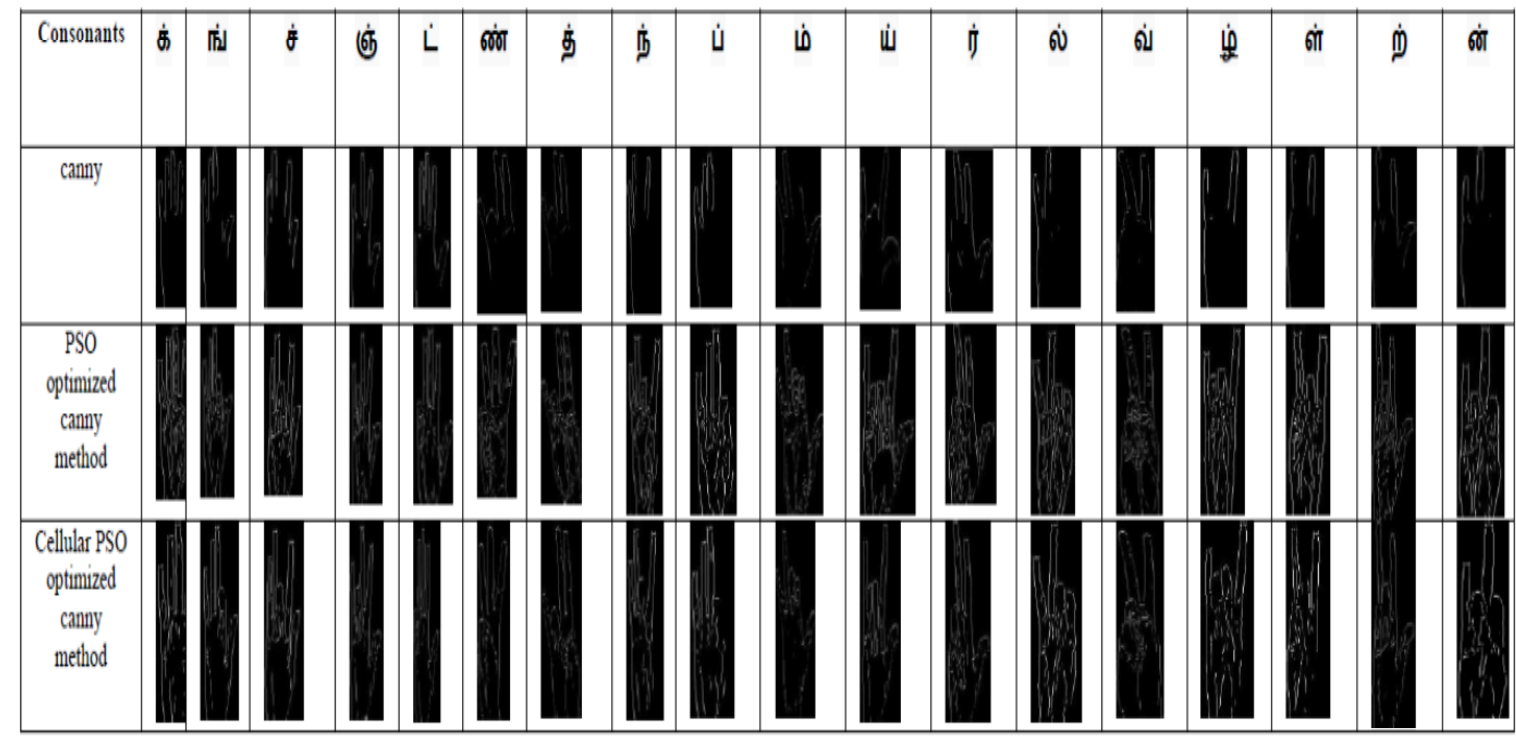

Figure 2. Visual Comparison of Canny and Optimized Canny with PSO and CPSO

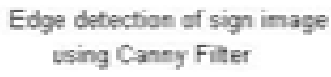

Edge detection of sign inase

using Canny Filter

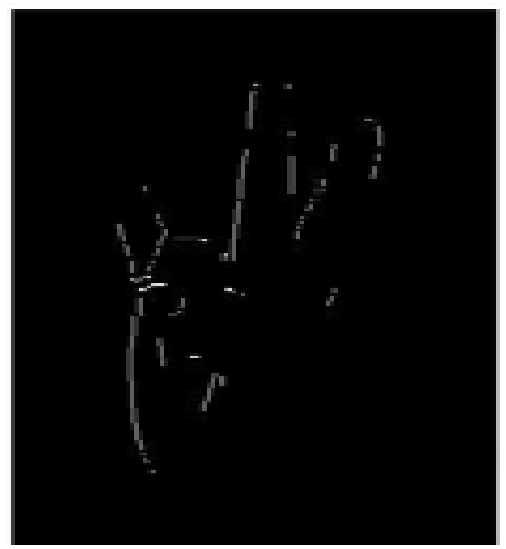

[a]
Edge detection of sign imspe using FSO Dptimized Ganm Fiter

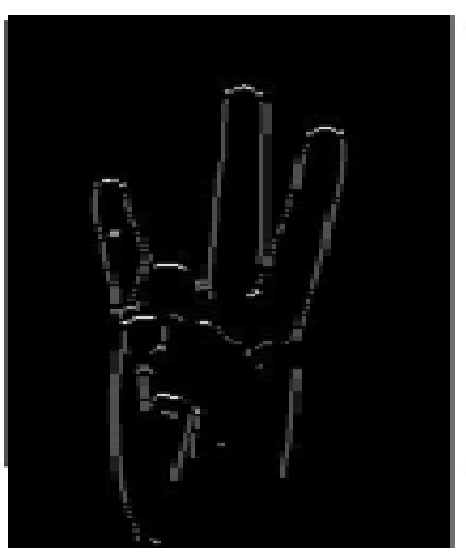

l]]
Edpe detection of sign imspe Geluar PSO Optimized Canny Fiter

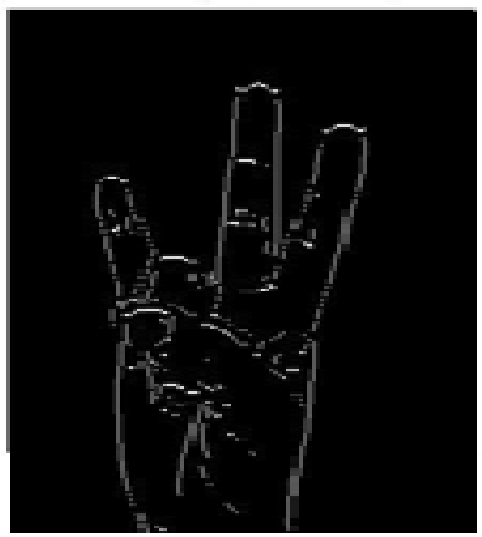

$[6]$

Figure 3. Edge detected from Hand Sign Image using Canny (a), PSO based Canny (b), and Cellular PSO based Canny (c)

\section{CONCLUSION}

Edge detection is the main segmentation process carried out in finding the boundary of the objects within the images. Canny edge detection is considered to the best edge detection method and it seems to produce false detection in noisy environment. An objective is proposed to develop an optimized edge detector that increases the localization accuracy of edge detection, especially in real time digital images. The goal of improved canny edges is achieved by introducing a hybrid optimization technique to find optimal threshold values which is a combination of PSO with cellular organism inspired from fibroblast. The proposed approach has improved the canny detector to connect the short edge contours into longer contours, and the experimental results validate its potentiality with different metrics. The results obtained will lead to higher accuracy of the classification method, adopted for the development of Tamil sign language recognition system. 
Table 1. Comparison of the the proposed method based on performance metrics

\begin{tabular}{|c|c|c|c|c|c|c|}
\hline \multirow{2}{*}{$\begin{array}{l}\text { Tamil constants } \\
\text { (Each sign with } 10 \\
\text { different signers) }\end{array}$} & \multicolumn{3}{|c|}{$\begin{array}{l}\text { Similarity Index } \\
\text { (mean) }\end{array}$} & \multicolumn{3}{|c|}{$\begin{array}{l}\text { Pearson correlation coefficient } \\
\text { (mean) }\end{array}$} \\
\hline & Canny & $\begin{array}{c}\text { PSO } \\
\text { optimized } \\
\text { canny } \\
\end{array}$ & $\begin{array}{c}\text { CPSO } \\
\text { optimized } \\
\text { canny }\end{array}$ & Canny & $\begin{array}{c}\text { PSO } \\
\text { optimized } \\
\text { canny }\end{array}$ & $\begin{array}{c}\text { CPSO } \\
\text { optimized } \\
\text { canny }\end{array}$ \\
\hline க் & 1.57503 & 1.57522 & 3.35514 & 0.7139 & 0.868 & 1.1186 \\
\hline ங் & 1.07352 & 1.07372 & 2.43706 & 0.4585 & 0.6082 & 0.7793 \\
\hline ச் & 1.44739 & 1.44764 & 3.2678 & 0.7026 & 0.8778 & 1.0844 \\
\hline ஞ் & 1.4929 & 1.49307 & 3.15721 & 0.5259 & 0.6666 & 0.8917 \\
\hline ட் & 1.21324 & 1.51163 & 3.74154 & 0.4583 & 0.5498 & 0.7342 \\
\hline ண் & 1.32979 & 1.44866 & 3.58511 & 0.7213 & 1.0103 & 1.458 \\
\hline த் & 1.43536 & 1.34258 & 3.44578 & 0.5948 & 0.8514 & 1.0131 \\
\hline ந் & 1.22323 & 1.28198 & 2.93116 & 0.6134 & 0.7649 & 1.0048 \\
\hline ப் & 1.10942 & 1.10961 & 2.41007 & 0.4802 & 0.6278 & 0.8325 \\
\hline ம் & 0.93203 & 0.9341 & 2.19098 & 0.3101 & 0.4441 & 0.6134 \\
\hline ய் & 1.05086 & 1.07765 & 2.70442 & 0.33 & 0.5148 & 0.8615 \\
\hline ர் & 1.02808 & 1.02829 & 2.35656 & 0.4201 & 0.5866 & 0.8375 \\
\hline ல் & 0.45689 & 0.45704 & 1.21129 & 0.3133 & 0.4491 & 0.5777 \\
\hline வ் & 1.09779 & 1.04162 & 2.10948 & 0.554 & 0.7892 & 1.1656 \\
\hline ழ் & 0.73188 & 0.73202 & 1.68165 & 0.3161 & 0.4448 & 0.6104 \\
\hline ஸ் & 0.78686 & 0.83769 & 2.08518 & 0.5021 & 0.7609 & 0.9824 \\
\hline 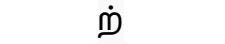 & 1.06134 & 1.06185 & 2.41274 & 0.4191 & 0.6127 & 0.8114 \\
\hline ன் & 1.12669 & 1.26164 & 2.57479 & 0.430366 & 0.7424 & 0.9927 \\
\hline
\end{tabular}

\section{ACKNOWLEDGEMENTS}

This work is supported by the project entitled "Investigation on view based methodological approaches for Tamil Sign language Recognition” (No. F. 30-42/2014 (BSR)) funded by UGC - BSR Research Start -Up- Grant.

\section{REFERENCES}

[1] P. Ghate, et al., "An Introduction to the Signing System for Indian Languages," Part II - Additional Signs. Bombay, Ali Yavar Jung National Institute for the Hearing Handicapped, 1990.

[2] M. Krishnaveni, et al., "Efficient Removal of Impulse Noise in Tamil Sign Language Digital Images using PSO Based Weighted Median Filter," International Journal of Applied Engineering Research (IJAER), vol. 10, pp. 40474-40480, 2015.

[3] N. Salman, "Image Segmentation Based on Watershed and Edge Detection Techniques," The International Arab Journal of Information Technology, vol. 3, pp. 104-110, 2006.

[4] S. Jansi and P. Subashini, "Optimized Adaptive Thresholding based Edge Detection Method for MRI Brain Images," International Journal of Computer Applications, vol. 51, pp. 1-8, 2012.

[5] J. Canny, "A Computational Approach to Edge Detection," IEEE Transaction on Pattern Analysis and Machine Intelligence, vol. 8, pp. 679-698, 1986.

[6] H. Pan, et al., "Particle Swarm Optimization for Function Optimization in Noisy Environment," Journal of Applied Mathematics and Computation, vol. 181, pp. 908-919, 2006.

[7] L. I. Hong-qi, et al., "An Improved PSO-based of Harmony Search for Complicated Optimization Problems," International Journal of Hybrid Information Technology, vol. 1, pp. 91-98, 2008.

[8] G. Coath and S. Halgamuge, "A Comparison of Constraint-Handling Methods for the Application of Particle Swarm Optimization to Constrained Nonlinear Optimization Problems," IEEE Congress on Evolutionary Computation, vol. 4, pp. 2419-2425, 2003.

[9] C. R. Eberhart and Y. Shi, "Particle Swarm Optimization: Developments, Applications and Resources," IEEE Xplore, vol. 1, pp. 81-86, 2001. doi: http://dx.doi.org/10.1109/CEC.2001.934374

[10] H. Stebbings, "Cell Motility,” Encyclopedia of Life Sciences, pp. 1-6, 2001.

[11] C. J. Dallon and A. J. Sherratt, "A Mathematical Model for Fibroblast and Collagen Orientation," Bulletin of Mathematical Biology, vol. 60, pp.101-129, 1998. 


\section{BIOGRAPHIES OF AUTHORS}

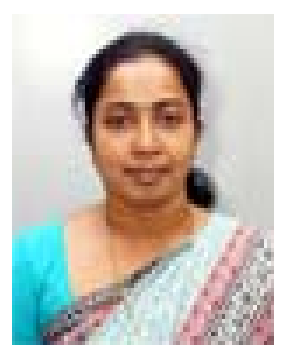

Dr. M. Krishnaveni is an Assistant Professor in the Department of Computer Science, Avinashilingam University for Women, Coimbatore. She has 7 years of research experience working as a researcher in Naval Research Board, Defence Research Development Organisation, Ministry of Defence. She was been a consultant for military related application project. She has published several papers in her research specialization with an overall count of 61 both nationally and internationally. She focuses on research and experimental processes with the use of digital technologies such as MATLAB, Computer Science, Biological computation along with machine intelligence and real-time physical computation. She also acts as a resource person and holds national and international funded research projects which come under University Grants Commission (India) and Department of Science and Technology.

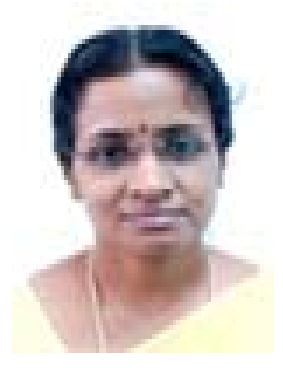

Dr. P. Subashini received a B.Sc. (Mathematics) Degree from Bharathiar University, Tamil Nadu, India in 1990 and M.C.A. Degree from the same University in 1993. She has also received Degrees of M.Phil. and Ph.D. respectively in Computer Science in the years 1998 and 2009 respectively from Avinashilingam University for Women, Tamil Nadu, India. From 1994 to 2007, she worked for the Department of Computer Science, Avinashilingam University for Women, where she is currently in the position of Professor. She has also held short-term appointments at several institutions around the State. She is a member of the editorial board of several international journals, and reviews several conferences. She has been invited as speaker to several workshops and organized international workshops and special sessions at conferences.

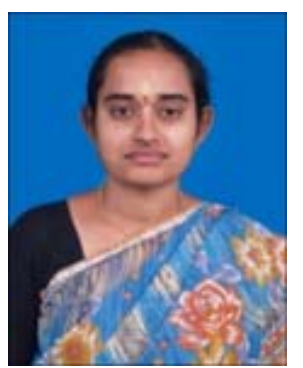

T. T. Dhivyaprabha has completed M.Sc., M.Phil. in Bharathiar University. She has 4 years of teaching experience. She is pursuing Ph.D. (Computer Science) programme in the Department of Computer Science, Avinashilingam University for Women, Coimbatore. Her areas of interest include Computational Intelligence, Optimization Technique and Image Processing. 